(C) The Authors 2012. The online version of this article is published within an Open Access environment subject to the conditions of the Creative Commons Attribution-NonCommercial-ShareAlike licence $<$ http://creativecommons.org/licenses/by-nc-sa/2.5/ $>$. The written permission of Cambridge University Press must be obtained for commercial re-use.

\title{
Trends in dietary energy, fat, carbohydrate and protein intake in Chinese children and adolescents from 1991 to 2009
}

\author{
Zhaohui Cui* and Michael J. Dibley \\ Sydney School of Public Health, Sydney Medical School, Room 307A, Edward Ford Building (A27), University of Sydney, \\ NSW 2006, Australia
}

(Submitted 15 June 2011 - Final revision received 14 November 2011 - Accepted 21 November 2011 - First published online 16 January 2012)

\begin{abstract}
Few studies have examined nutrition transition in children in China. Our aim, in the present study, was to examine temporal trends in dietary energy, fat, carbohydrate and protein intake in Chinese children aged 7-17 years. The analysis used individual level, consecutive $3 \mathrm{~d}$ dietary recall data from seven rounds of the China Health and Nutrition Surveys in 1991 ( $n$ 2714), 1993 ( $n$ 2542), 1997 ( $n$ 2516), 2000 ( $n$ 2142), 2004 ( $n$ 1341), 2006 ( $n$ 1072) and 2009 ( $n$ 996). Mixed-effect models were constructed to obtain adjusted means and to examine trends after adjusting for intra-class correlation within clusters and for covariates including age, sex, urban/rural residence and income. From 1991 to 2009 , daily energy intake steadily declined from $9511 \cdot 0$ to $7658.2 \mathrm{~kJ}(P<0 \cdot 0001)$. There was a steady decline in daily carbohydrate intake from 382.5 to $254.1 \mathrm{~g}(P<0.0001)$, and in the proportion of energy from carbohydrate from 66.7 to $56.8 \%$ $(P<0.0001)$. In contrast, daily fat intake steadily increased from 54.8 to $66.0 \mathrm{~g}(P<0.0001)$, as did the proportion of energy from fat from 21.5 to $30.0 \%(P<0.0001)$. The proportion of children who consumed a diet with more than $30 \%$ of energy from fat increased from 20.1 to $49.4 \%(P<0.0001)$. The proportion of energy from protein increased from $11 \cdot 8$ to $13.1 \%(P<0 \cdot 0001)$, although daily protein intake dropped from 66.2 to $58.0 \mathrm{~g}(P<0 \cdot 0001)$. Our data suggest that Chinese children have been undergoing a rapid nutrition transition to a high-fat diet.
\end{abstract}

\section{Key words: Children and adolescents: Nutrition transition: Macronutrients: China}

The world has been undergoing a profound nutrition transition associated with increased nutrition-related chronic noncommunicable diseases since the Second World War ${ }^{(1)}$. China, with the fastest economic growth in the last two decades in the world, has been undergoing a unique nutrition transition ${ }^{(2)}$ and a related steady increase in child obesity ${ }^{(3)}$. To tailor effective interventions to address child obesity in China, it is necessary to understand the transition in dietary patterns, which is one side of energy balance, in Chinese children.

Most previous studies ${ }^{(1,2,4,5)}$ examining this nutrition transition have been conducted in adults. There have been some reports of changes in macronutrient intake in US youth over several decades ${ }^{(6-10)}$. However, few studies from the developing world, including China, have examined trends in dietary patterns in children even though childhood and adolescence is a critical period for the establishment of a healthy lifestyle and the prevention of obesity and other nutrition-related non-communicable chronic diseases ${ }^{(6)}$. The nutrition transition in US children with a decline in the proportion of energy from fat between the 1960s and the 1990s may not be applicable to Chinese children because of the low fat content in traditional Chinese diets and the demonstrated differences in nutrition transition between Chinese and American adults ${ }^{(5,11)}$.

The examination of a nutrition transition is often constrained by the lack of detailed nationwide individual food intake data in most developing countries ${ }^{(12-17)}$. Substituting individual consumption data with ecological nutrient intake data to examine a nutrition transition is problematic, because an association observed between variables at a group level may not represent an association that exists at an individual level $^{(18,19)}$. Also, changes in dietary measurement methods between surveys conducted over many years apart make the interpretation of trends in dietary intake difficult ${ }^{(9,19)}$. The purpose of the present study was to examine the temporal trends in dietary energy, fat, carbohydrate and protein intake in Chinese children in the last two decades using the China Health and Nutrition Surveys (CHNS), in which individual level, consecutive $3 \mathrm{~d}$ dietary recall data were collected using the same methods in each survey.

Abbreviations: CHNS, China Health and Nutrition Surveys; RMB, renminbi. 


\section{Subjects and methods}

\section{Study design}

The China Health and Nutrition Surveys are an ongoing series of longitudinal household surveys conducted in eight provinces from 1989 to 1997, and expanded to nine provinces since 2000, from all thirty-one provinces (or regions) of mainland China. These provinces varied substantially in geography, economic development and health indicators ${ }^{(20)}$. When Liaoning province was unable to continue to participate in 1997, a new province (Heilongjiang) from the same region of China was added. However, the dropped province returned to the study in 2000. So far, there have been eight rounds of surveys conducted in 1989, 1991, 1993, 1997, 2000, 2004, 2006 and 2009. The sampling procedure has been described in detail elsewhere $^{(20)}$, and comprised a multistage random-cluster sample selected in each province. Firstly, four counties (one low-, two middle- and one high-income county, defined on the basis of per-capita income) were randomly selected from each province using a weighted sampling method. In addition, the provincial capital and a low-income city were selected when feasible. Then, the township capital and three villages within the counties and urban and suburban neighbourhoods within the cities were randomly sampled, and twenty households were randomly selected within each neighbourhood. All individuals in each household were interviewed.

This study was conducted according to the guidelines laid down in the Declaration of Helsinki. Survey protocols, instruments and the process for obtaining informed consent for this study were approved by institutional review committees of the University of North Carolina at Chapel Hill and the National Institute for Nutrition and Food Safety, China Center for Disease Control and Prevention. Written informed consent was obtained from all subjects.

\section{Participants}

In the eight provinces that participated in the 1991 survey, participants aged 7-17 years were included in the analysis. Heilongjiang was included in 1997 to ensure adequate geographical coverage of China, but was then excluded in the following surveys once Liaoning had returned, to ensure that the provinces in each survey were from a comparable variety of regions in China.

\section{Socio-demographic data}

Demographic information included the child's date of birth, sex, residence and annual family income. Per-capita annual family income in renminbi (RMB) was obtained by dividing annual family income by household size. The per-capita annual family income in each survey was inflated to values in 2009 by adjusting for consumer price index and then categorised into tertiles as high, medium and low income by urban and rural areas.

\section{Dietary data}

Household food consumption data and individual dietary recall data were collected during three consecutive days that were randomly allocated from Monday to Sunday and almost equally balanced across the $7 \mathrm{~d}$ of the week for each sampling unit. Household food consumption was determined using a weighing technique. All foods, including edible oils, remaining after the last meal before initiation of the survey were weighed and recorded. During the three survey days, whenever foods were brought into the household unit, they were weighed, and preparation waste was estimated when weighing was not possible. At the end of the survey, all remaining foods were again weighed and recorded. The foods eaten outside were not weighed. The number and personal characteristics of all household members and visitors were recorded at each meal.

Individual dietary intake data were collected by asking each household member to report regarding all food consumed away from home and at home on a $24 \mathrm{~h}$-recall basis except for young children under 12 years of age, whose mother or caregiver who handled food preparation in the household was asked to recall the child's consumption. Using food models and picture aids, trained field interviewers

Table 1. Characteristics of participants aged 7-17 years from 1991 to 2009 in China Health and Nutrition Surveys (Number of participants, percentages or mean values with their standard errors)

\begin{tabular}{lrrrrrrr}
\hline & 1991 & 1993 & 1997 & 2000 & 2004 & 2006 & 2009 \\
\hline Sample (n) & 2714 & 2542 & 2516 & 2142 & 1341 & 1072 & 996 \\
Age (years) & & & & & & & \\
$\quad$ Mean & $12 \cdot 5$ & $12 \cdot 2$ & $12 \cdot 1$ & $12 \cdot 7$ & $12 \cdot 7$ & $12 \cdot 2$ & $12 \cdot 0$ \\
$\quad$ SE & $0 \cdot 1$ & $0 \cdot 1$ & $0 \cdot 1$ & $0 \cdot 1$ & $0 \cdot 1$ & $0 \cdot 1$ & $0 \cdot 1$ \\
Male (\%) & $51 \cdot 5$ & $51 \cdot 6$ & $53 \cdot 5$ & $53 \cdot 3$ & $52 \cdot 3$ & $53 \cdot 5$ & $54 \cdot 8$ \\
Urban (\%) & $25 \cdot 6$ & $25 \cdot 3$ & $29 \cdot 2$ & $27 \cdot 5$ & $28 \cdot 1$ & $29 \cdot 0$ & $26 \cdot 9$ \\
$\begin{array}{l}\text { Income (RMB) } \\
\quad \text { Urban }\end{array}$ & & & & & & & \\
$\quad$ Mean & 3280 & 3988 & 4325 & 5661 & 6964 & 8658 & 10566 \\
$\quad$ SE & 75 & 125 & 113 & 267 & 344 & 506 & 565 \\
$\quad$ Rural & & & & & & & \\
$\quad$ Mean & 2060 & 2289 & 3106 & 3836 & 4654 & 5281 & 7935 \\
$\quad$ SE & 35 & 43 & 63 & 85 & 150 & 276 & 401 \\
\hline
\end{tabular}

RMB, renminbi.

${ }^{\star \star \star}$ Significant trend across the survey years $(P<0.0001$; test for trend). 
recorded the types, amounts, type of meal, and place of consumption of all food items during the previous day. Respondents were prompted about snacks and shared dishes. Housewives and other household members were encouraged to provide additional information to determine the amounts of particular food items in dishes consumed in the household. Cooking oil from the household food consumption data was used to supplement the individual dietary intake data ${ }^{(21)}$. The quality of the data collection was checked by comparing an individual's average daily dietary intake, calculated from the household survey and individual dietary intake based on $24 \mathrm{~h}$-recall data, respectively. Where significant discrepancies were found, the household and the individual in question were revisited and asked about their food consumption to resolve these discrepancies. In a few cases, subjects had missed $1 \mathrm{~d}$ because of absence, but over $99 \%$ of the sample was available for the full $3 \mathrm{~d}$ of data collection.

All field workers were trained nutritionists who are otherwise professionally engaged in nutrition work in their own counties and who have participated in other national surveys. Almost all interviewers were graduates of post-secondary schools; many had 4-year degrees. In addition, all interviewers were trained for at least $3 \mathrm{~d}$ in the collection of dietary data in each survey.

The 1991 Food Composition Table for China was utilised to calculate nutrient values for the dietary data of 2000 and previous years. A newer version of Food Composition Table (2002) was used for the 2004 survey and the latest version (2004) was used for the 2006 and 2009 surveys.

\section{Statistical analysis}

Adjusted means and standard errors were used to describe the distribution of continuous variables after adjusting for complex sampling and covariates including age, sex, urban/ rural residence and per-capita annual family income tertiles. Age was adjusted as a continuous variable, and income as a categorical variable. Mixed-effect models were used to calculate adjusted mean values of dietary energy, fat, carbohydrate, protein and the percentages of their energy contributions in the total energy intake and to examine the temporal trends after adjusting for intra-class correlation within clusters and covariates including age, sex, urban/rural residence and income level. All the analyses were conducted using SAS (version 9; SAS Institute).

\section{Results}

Table 1 presents the characteristics of the participant children aged 7-17 years in the CHNS by survey year. The sample size was 2714 in 1991, 2542 in 1993, 2516 in 1997, 2142 in 2000, 1341 in 2004, 1072 in 2006 and 996 in 2009, respectively. The mean ages ranged from $12 \cdot 0$ to $12 \cdot 7$ years, and even though these differences between the survey years were small, they were statistically significant $(P<0 \cdot 0001)$. There were no significant differences in the distribution of sex and urban/rural residence across the survey years. The inflationadjusted per-capita annual family income increased from $3280 \mathrm{RMB}$ in 1991 to $10566 \mathrm{RMB}$ in 2009 in urban areas $(P<0.0001)$, far larger than the increase in rural areas from 2060 RMB to 7935 RMB during the same period $(P<0 \cdot 0001)$.

As shown in Table 2, energy intake among children steadily declined in different age ( $7-10,11-13$ and $14-17$ years), sex, urban/rural residence and income groups $(P<0 \cdot 0001)$. The average daily energy intake decreased from $9511.0 \mathrm{~kJ}$ in 1991 to $7658.2 \mathrm{~kJ}$ in 2009 . It is worth noting that the decline in energy intake in rural children $(2030 \cdot 0 \mathrm{~kJ} / \mathrm{d})$ was larger than that in urban children $(1225.9 \mathrm{~kJ} / \mathrm{d})$. Further, the largest

Table 2. Daily energy intake (kJ), by age, sex, residence and income, in Chinese children from 1991 to $2009^{\star \star \star} \dagger$

(Mean values with their standard errors)

\begin{tabular}{|c|c|c|c|c|c|c|c|c|c|c|c|c|c|c|}
\hline & \multicolumn{2}{|c|}{1991} & \multicolumn{2}{|c|}{1993} & \multicolumn{2}{|c|}{1997} & \multicolumn{2}{|c|}{2000} & \multicolumn{2}{|c|}{2004} & \multicolumn{2}{|c|}{2006} & \multicolumn{2}{|c|}{2009} \\
\hline & Mean & SE & Mean & SE & Mean & SE & Mean & SE & Mean & SE & Mean & SE & Mean & SE \\
\hline All & 9511.0 & $133 \cdot 1$ & $9114 \cdot 2$ & $133 \cdot 8$ & $8310 \cdot 4$ & $132 \cdot 8$ & $8594 \cdot 4$ & $135 \cdot 7$ & $8129 \cdot 2$ & $143 \cdot 7$ & $7630 \cdot 1$ & $148 \cdot 8$ & $7658 \cdot 2$ & $150 \cdot 9$ \\
\hline \multicolumn{15}{|c|}{ Age group (years) } \\
\hline $7-10$ & 8208.5 & 163.5 & $7945 \cdot 9$ & 163.7 & 7185.5 & $161 \cdot 1$ & 7433.9 & $180 \cdot 6$ & 7053.1 & $195 \cdot 6$ & $6549 \cdot 0$ & $194 \cdot 7$ & $6876 \cdot 5$ & 196.9 \\
\hline $11-13$ & $9540 \cdot 3$ & $168 \cdot 6$ & $9189 \cdot 9$ & 169.5 & $8482 \cdot 0$ & $167 \cdot 1$ & $8711 \cdot 0$ & $166 \cdot 1$ & $8389 \cdot 1$ & $195 \cdot 2$ & $7962 \cdot 9$ & $207 \cdot 8$ & $7613 \cdot 1$ & 204.5 \\
\hline $14-17$ & $10760 \cdot 0$ & 174.3 & 10264.0 & $177 \cdot 7$ & 9311.4 & 178.5 & 9664.9 & $180 \cdot 5$ & $9130 \cdot 8$ & $192 \cdot 1$ & 8655.6 & $215 \cdot 6$ & $8478 \cdot 4$ & 228.0 \\
\hline \multicolumn{15}{|l|}{ Sex } \\
\hline Male & 9885.0 & $138 \cdot 1$ & $9516 \cdot 1$ & $139 \cdot 1$ & 8663.7 & $136 \cdot 8$ & 8978.3 & 141.9 & $8636 \cdot 4$ & $156 \cdot 9$ & 8083.2 & $165 \cdot 1$ & 8131.5 & $166 \cdot 7$ \\
\hline Female & 9005.5 & $165 \cdot 9$ & $8631 \cdot 7$ & $167 \cdot 2$ & $7860 \cdot 7$ & $166 \cdot 6$ & $8120 \cdot 6$ & $171 \cdot 8$ & $7560 \cdot 4$ & $184 \cdot 8$ & $7132 \cdot 4$ & $194 \cdot 6$ & 7097.5 & 198.7 \\
\hline \multicolumn{15}{|c|}{ Urban income } \\
\hline Low & 8811.0 & $248 \cdot 4$ & $8656 \cdot 0$ & $251 \cdot 6$ & 8495.5 & 241.0 & $8580 \cdot 9$ & 259.2 & $7717 \cdot 0$ & $290 \cdot 1$ & $7197 \cdot 8$ & $308 \cdot 1$ & $7329 \cdot 0$ & 320.5 \\
\hline Medium & $9203 \cdot 6$ & 233.3 & 8677.5 & 241.3 & $8820 \cdot 3$ & 228.0 & $8409 \cdot 6$ & $246 \cdot 3$ & $8561 \cdot 3$ & $282 \cdot 1$ & $7765 \cdot 1$ & $301 \cdot 6$ & $8227 \cdot 0$ & 314.4 \\
\hline High & $8996 \cdot 7$ & 253.0 & 9051.6 & $256 \cdot 0$ & $9017 \cdot 4$ & 246.7 & $8875 \cdot 3$ & $258 \cdot 1$ & $8444 \cdot 2$ & $292 \cdot 3$ & 8288.0 & 304.6 & $8996 \cdot 7$ & 253.0 \\
\hline All & $9026 \cdot 9$ & $195 \cdot 3$ & $8815 \cdot 7$ & $197 \cdot 2$ & $8797 \cdot 7$ & $192 \cdot 1$ & $8662 \cdot 6$ & $198 \cdot 2$ & 8223.6 & $212 \cdot 3$ & $7742 \cdot 1$ & $219 \cdot 8$ & $7801 \cdot 0$ & 226.8 \\
\hline \multicolumn{15}{|c|}{ Rural income } \\
\hline Low & $9530 \cdot 2$ & $190 \cdot 2$ & $9026 \cdot 2$ & $190 \cdot 8$ & 7919.7 & $192 \cdot 2$ & $8019 \cdot 1$ & 197.4 & $7344 \cdot 0$ & $217 \cdot 3$ & $7572 \cdot 8$ & $230 \cdot 1$ & 6794.9 & 236.1 \\
\hline Medium & 9498.9 & $186 \cdot 4$ & $9190 \cdot 5$ & $187 \cdot 6$ & $8005 \cdot 7$ & 187.5 & $8562 \cdot 8$ & $195 \cdot 1$ & 8361.5 & 216.9 & $7342 \cdot 0$ & 231.5 & $7366 \cdot 2$ & 233.5 \\
\hline High & 9763.4 & $213 \cdot 2$ & 9304.9 & $216 \cdot 2$ & $8300 \cdot 4$ & $215 \cdot 0$ & $9045 \cdot 4$ & $225 \cdot 0$ & $8624 \cdot 1$ & $253 \cdot 3$ & 7983.5 & $272 \cdot 2$ & 8531.8 & 272.5 \\
\hline All & 9653.6 & 155.6 & $9182 \cdot 7$ & $156 \cdot 5$ & $8078 \cdot 2$ & $156 \cdot 1$ & 8535.1 & $159 \cdot 6$ & $8109 \cdot 9$ & $169 \cdot 9$ & $7612 \cdot 0$ & $177 \cdot 0$ & $7623 \cdot 6$ & $178 \cdot 6$ \\
\hline
\end{tabular}

${ }^{\star \star \star}$ Significant trend in each subgroup across the survey years $(P<0.0001$; test for trend).

$\dagger$ Values adjusted for age, sex, urban/rural residence and income. 
Table 3. Daily carbohydrate intake (g), by age, sex, residence and income, in Chinese children from 1991 to $2009^{\star \star \star} \dagger$ (Mean values with their standard errors)

\begin{tabular}{|c|c|c|c|c|c|c|c|c|c|c|c|c|c|c|}
\hline & \multicolumn{2}{|c|}{1991} & \multicolumn{2}{|c|}{1993} & \multicolumn{2}{|c|}{1997} & \multicolumn{2}{|c|}{2000} & \multicolumn{2}{|c|}{2004} & \multicolumn{2}{|c|}{2006} & \multicolumn{2}{|c|}{2009} \\
\hline & Mean & SE & Mean & SE & Mean & SE & Mean & SE & Mean & SE & Mean & SE & Mean & SE \\
\hline All & 382.5 & 8.5 & $357 \cdot 0$ & 8.5 & 311.0 & 8.4 & 300.9 & 8.5 & 282.5 & 8.7 & $270 \cdot 6$ & $8 \cdot 8$ & $254 \cdot 1$ & 8.8 \\
\hline \multicolumn{15}{|c|}{ Age group (years) } \\
\hline $7-10$ & 325.6 & $7 \cdot 8$ & $305 \cdot 6$ & 7.8 & 264.9 & $7 \cdot 8$ & 254.7 & 8.2 & $244 \cdot 0$ & 8.5 & $226 \cdot 5$ & 8.5 & $218 \cdot 5$ & 8.5 \\
\hline $11-13$ & 384.9 & $9 \cdot 1$ & 362.5 & 9.1 & 317.4 & $9 \cdot 0$ & 308.5 & 9.0 & 293.0 & $10 \cdot 0$ & 288.5 & $10 \cdot 4$ & $258 \cdot 0$ & $10 \cdot 3$ \\
\hline $14-17$ & 433.7 & $10 \cdot 8$ & 402.9 & 10.9 & 353.7 & $10 \cdot 8$ & 343.5 & $10 \cdot 9$ & $316 \cdot 4$ & $11 \cdot 2$ & $308 \cdot 3$ & $11 \cdot 8$ & $289 \cdot 7$ & $12 \cdot 2$ \\
\hline \multicolumn{15}{|l|}{ Sex } \\
\hline Male & 397.3 & 8.7 & 373.5 & 8.7 & 325.5 & 8.6 & 315.9 & 8.7 & 298.5 & $9 \cdot 1$ & $289 \cdot 0$ & 9.4 & 273.7 & 9.4 \\
\hline Female & $362 \cdot 7$ & 8.6 & $337 \cdot 2$ & 8.6 & 293.9 & 8.6 & 284.8 & 8.7 & 263.4 & 9.0 & $251 \cdot 4$ & $9 \cdot 2$ & $233 \cdot 0$ & 9.3 \\
\hline \multicolumn{15}{|c|}{ Urban income } \\
\hline Low & 337.6 & $12 \cdot 3$ & $324 \cdot 2$ & $12 \cdot 4$ & $296 \cdot 7$ & $11 \cdot 8$ & 271.2 & $12 \cdot 4$ & $259 \cdot 2$ & 13.4 & $234 \cdot 1$ & 14.0 & $228 \cdot 0$ & 14.4 \\
\hline Medium & $327 \cdot 2$ & 9.4 & 294.5 & 9.7 & 288.5 & $9 \cdot 1$ & 253.7 & 9.6 & $266 \cdot 1$ & $10 \cdot 7$ & $247 \cdot 1$ & 11.4 & 245.5 & 11.8 \\
\hline High & 321.9 & 7.9 & $295 \cdot 6$ & 8.0 & 284.7 & $7 \cdot 7$ & $271 \cdot 2$ & 8.1 & 262.6 & 9.5 & 245.4 & $10 \cdot 0$ & $243 \cdot 3$ & $10 \cdot 4$ \\
\hline All & 329.6 & 8.4 & 303.5 & $8 \cdot 4$ & $289 \cdot 7$ & 8.2 & 264.0 & 8.3 & $263 \cdot 2$ & 8.8 & 241.5 & $9 \cdot 0$ & 237.0 & $9 \cdot 2$ \\
\hline \multicolumn{15}{|c|}{ Rural income } \\
\hline Low & 402.2 & $11 \cdot 2$ & 375.5 & $11 \cdot 2$ & $312 \cdot 7$ & $11 \cdot 3$ & 303.6 & 11.5 & 268.6 & $12 \cdot 1$ & 287.6 & $12 \cdot 5$ & $242 \cdot 1$ & $12 \cdot 8$ \\
\hline Medium & $392 \cdot 1$ & $10 \cdot 7$ & 373.7 & $10 \cdot 7$ & $309 \cdot 2$ & $10 \cdot 7$ & 312.6 & $10 \cdot 9$ & $299 \cdot 1$ & 11.6 & $270 \cdot 2$ & $12 \cdot 1$ & 260.5 & $12 \cdot 2$ \\
\hline High & 391.6 & 10.5 & 362.5 & 10.6 & 318.0 & 10.5 & 316.9 & $10 \cdot 7$ & $296 \cdot 1$ & $11 \cdot 3$ & 284.6 & $11 \cdot 7$ & 268.9 & 11.7 \\
\hline All & 397.4 & $10 \cdot 1$ & 371.3 & $10 \cdot 1$ & $313 \cdot 1$ & $10 \cdot 0$ & $310 \cdot 3$ & $10 \cdot 1$ & 287.9 & $10 \cdot 3$ & $280 \cdot 2$ & 10.5 & $258 \cdot 7$ & 10.5 \\
\hline
\end{tabular}

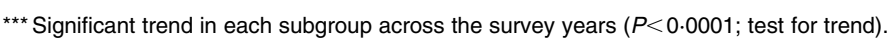

† Values adjusted for age, sex, urban/rural residence and income.

decline of $2735 \cdot 3 \mathrm{~kJ} / \mathrm{d}$ in energy intake was found in those from low-income rural families. This decline was larger than that in medium-income families $(2132 \cdot 7 \mathrm{~kJ} / \mathrm{d})$ or high-income families $(1231.6 \mathrm{~kJ} / \mathrm{d})$ in rural areas.

Similarly, as seen in Table 3, daily carbohydrate intake steadily dropped across the survey years in each age group, sex, urban/rural residence and different income groups $(P<0.0001)$. Daily carbohydrate intake dropped from $382.5 \mathrm{~g}$ in 1991 to $254.1 \mathrm{~g}$ in 2009 . The decline in carbohydrate intake in rural children was higher than that in urban children. Further, the decline in carbohydrate intake in children from lower-income families was higher than that in those from higher-income families in both urban and rural areas. As a result, the proportion of energy from carbohydrate decreased from $66.7 \%$ in 1991 to $56.8 \%$ in 2009 ( $P<0.0001$, data not shown). Similar trends were found in both urban and rural areas as shown in Fig. 1

Table 4 shows that daily fat intake in Chinese children from 1991 to 2009 increased during this period from 54.8 to $66 \cdot 0 \mathrm{~g}$ $(P<0.0001)$. As a result, the proportion of energy from fat increased from $21.5 \%$ in 1991 to $30.0 \%$ in 2009, and even reached $34.6 \%$ in urban areas $(P<0.0001)$, as shown in Fig. 1. The proportion of children who consumed a diet with more than $30 \%$ of energy from fat increased from $20 \cdot 1 \%$ in 1991 to $49 \cdot 4 \%$ in 2009 (Table 5). Further, the increase in the proportion of children who consumed more than $30 \%$ (a)

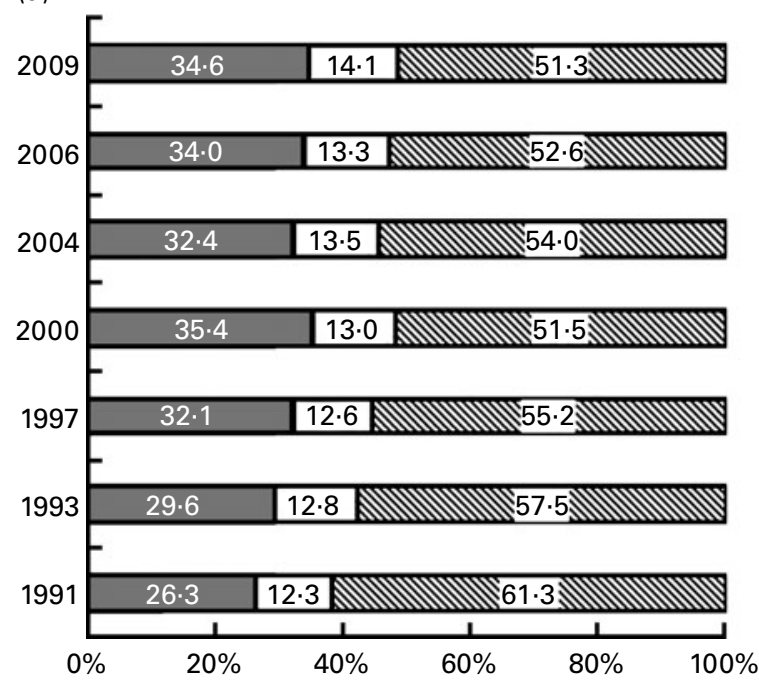

(b)

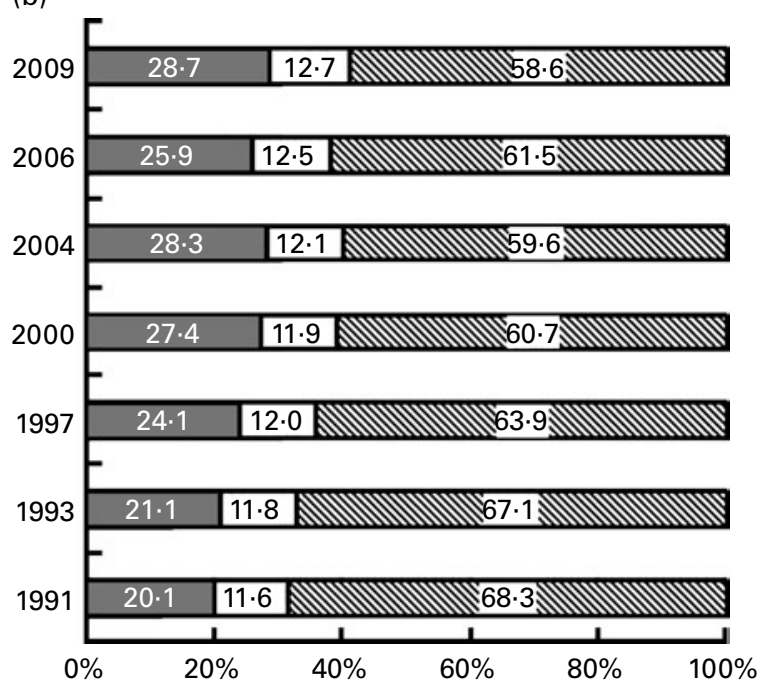

Fig. 1. Percentages of energy from protein $(\square)$, carbohydrate $(\square)$ and fat $(\square)$ in Chinese children from 1991 to 2006 by residence: (a) urban; (b) rural. Proportions were adjusted for age, sex, urban/rural residence and income. There was a significant trend in the proportions of energy from fat, carbohydrate and protein across the survey years $(P<0.0001$; test for trend). 
Table 4. Daily fat intake (g), by age, sex, residence and income, in Chinese children from 1991 to $2009 \dagger$

(Mean values with their standard errors)

\begin{tabular}{|c|c|c|c|c|c|c|c|c|c|c|c|c|c|c|}
\hline & \multicolumn{2}{|c|}{1991} & \multicolumn{2}{|c|}{1993} & \multicolumn{2}{|c|}{1997} & \multicolumn{2}{|c|}{2000} & \multicolumn{2}{|c|}{2004} & \multicolumn{2}{|c|}{2006} & \multicolumn{2}{|c|}{2009} \\
\hline & Mean & SE & Mean & SE & Mean & SE & Mean & SE & Mean & SE & Mean & SE & Mean & SE \\
\hline$A l^{* \star \star}$ & $54 \cdot 8$ & 2.9 & $56 \cdot 4$ & 2.9 & $57 \cdot 3$ & 2.9 & $68 \cdot 6$ & 2.9 & 64.9 & $3 \cdot 0$ & $58 \cdot 0$ & $3 \cdot 1$ & $66 \cdot 0$ & $3 \cdot 1$ \\
\hline \multicolumn{15}{|c|}{ Age group (years) } \\
\hline $7-10^{\star \star \star}$ & $48 \cdot 3$ & 2.9 & $50 \cdot 5$ & $2 \cdot 9$ & $50 \cdot 2$ & 2.9 & $60 \cdot 2$ & $3 \cdot 3$ & 55.9 & $3 \cdot 6$ & $51 \cdot 6$ & 3.5 & $63 \cdot 1$ & $3 \cdot 6$ \\
\hline $11-13^{\star \star \star}$ & $54 \cdot 4$ & $2 \cdot 7$ & $55 \cdot 5$ & $2 \cdot 7$ & $58 \cdot 3$ & $2 \cdot 6$ & $67 \cdot 9$ & 2.6 & $66 \cdot 4$ & 2.9 & $57 \cdot 7$ & $3 \cdot 0$ & $62 \cdot 9$ & $3 \cdot 0$ \\
\hline $14-17^{\star \star \star}$ & $60 \cdot 7$ & $3 \cdot 1$ & $62 \cdot 1$ & $3 \cdot 1$ & $61 \cdot 0$ & $3 \cdot 1$ & 74.4 & $3 \cdot 1$ & $72 \cdot 4$ & $3 \cdot 2$ & 63.6 & 3.5 & $68 \cdot 0$ & $3 \cdot 6$ \\
\hline \multicolumn{15}{|l|}{ Sex } \\
\hline Male ${ }^{\star \star \star}$ & $56 \cdot 7$ & $3 \cdot 0$ & $57 \cdot 9$ & $3 \cdot 1$ & $59 \cdot 0$ & $3 \cdot 0$ & 70.5 & $3 \cdot 1$ & $69 \cdot 3$ & $3 \cdot 2$ & $59 \cdot 9$ & $3 \cdot 2$ & $67 \cdot 6$ & $3 \cdot 2$ \\
\hline Female ${ }^{\star \star \star}$ & $51 \cdot 1$ & $2 \cdot 6$ & $53 \cdot 4$ & $2 \cdot 6$ & $53 \cdot 6$ & $2 \cdot 6$ & $64 \cdot 1$ & $2 \cdot 7$ & $59 \cdot 6$ & 2.9 & $54 \cdot 2$ & $3 \cdot 1$ & $62 \cdot 1$ & $3 \cdot 2$ \\
\hline \multicolumn{15}{|l|}{ Urban } \\
\hline Low $^{\star *}$ & $57 \cdot 3$ & $4 \cdot 3$ & $59 \cdot 5$ & $4 \cdot 3$ & $66 \cdot 4$ & $4 \cdot 1$ & 79.5 & $4 \cdot 3$ & 62.5 & $4 \cdot 7$ & 61.9 & $4 \cdot 8$ & $68 \cdot 1$ & $5 \cdot 0$ \\
\hline Medium* & 67.9 & $4 \cdot 4$ & $68 \cdot 6$ & 4.4 & 75.9 & $4 \cdot 2$ & $81 \cdot 0$ & $4 \cdot 4$ & $78 \cdot 7$ & $5 \cdot 0$ & 68.5 & $5 \cdot 3$ & $78 \cdot 8$ & $5 \cdot 4$ \\
\hline High & 65.5 & 4.4 & $78 \cdot 4$ & 4.4 & 82.5 & $4 \cdot 3$ & 83.5 & $4 \cdot 4$ & $75 \cdot 7$ & $4 \cdot 8$ & $80 \cdot 8$ & $5 \cdot 0$ & $68 \cdot 6$ & $5 \cdot 1$ \\
\hline All ${ }^{* * \star}$ & $64 \cdot 2$ & 3.9 & $69 \cdot 9$ & 3.9 & $75 \cdot 7$ & $3 \cdot 8$ & $83 \cdot 3$ & 3.9 & $72 \cdot 3$ & $4 \cdot 0$ & $71 \cdot 2$ & $4 \cdot 1$ & $73 \cdot 2$ & $4 \cdot 2$ \\
\hline \multicolumn{15}{|l|}{ Rural } \\
\hline $\operatorname{Low}^{\star \star *}$ & $46 \cdot 0$ & $2 \cdot 6$ & $45 \cdot 9$ & $2 \cdot 6$ & $47 \cdot 2$ & 2.6 & $54 \cdot 7$ & $2 \cdot 6$ & $53 \cdot 1$ & $2 \cdot 8$ & $49 \cdot 7$ & 2.9 & $50 \cdot 4$ & 3.0 \\
\hline 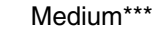 & $49 \cdot 2$ & $3 \cdot 3$ & $49 \cdot 9$ & $3 \cdot 3$ & $50 \cdot 2$ & $3 \cdot 3$ & $62 \cdot 7$ & 3.4 & $63 \cdot 2$ & 3.5 & $51 \cdot 0$ & $3 \cdot 6$ & $55 \cdot 4$ & $3 \cdot 7$ \\
\hline High $^{\star \star \star}$ & $58 \cdot 0$ & 3.4 & $59 \cdot 3$ & 3.5 & 53.9 & 3.5 & 71.6 & 3.7 & $71 \cdot 2$ & $4 \cdot 3$ & $59 \cdot 1$ & $4 \cdot 8$ & $82 \cdot 0$ & $4 \cdot 8$ \\
\hline All ${ }^{* \star *}$ & $52 \cdot 0$ & 2.9 & $52 \cdot 2$ & 2.9 & $51 \cdot 2$ & 2.9 & 63.7 & 2.9 & $63 \cdot 1$ & $3 \cdot 1$ & 54.0 & $3 \cdot 2$ & $64 \cdot 2$ & $3 \cdot 2$ \\
\hline
\end{tabular}

Significant trend across the survey years: ${ }^{\star} P<0.05,{ }^{\star \star} P<0.01,{ }^{\star \star \star} P<0.0001$; test for trend.

$\dagger$ Values adjusted for age, sex, urban/rural residence and income.

of energy from fat was greater in the lower-income group than in the higher-income group in urban areas, while the increase was larger in the higher-income group than in the lowerincome group in rural areas.

Table 6 presents the daily protein intake among Chinese children from 1991 to 2006, which steadily declined from 66.2 to $58.0 \mathrm{~g}$ during this period $(P<0.0001)$. In urban areas, there were no significant changes in the protein intake in children from each income group, while in rural areas the decline in protein intake in the lower-income group was larger than that in the higher-income group.

\section{Discussion}

Our present study indicates that energy intake dramatically decreased when the dietary pattern shifted from high carbohydrate to high fat. The proportion of children who consumed a high-fat diet more than doubled. These findings indicate that Chinese children have been undergoing a rapid nutrition transition over the last two decades as China has been undergoing rapid economic development. It is worth noting that the largest transitions in dietary patterns were found in children from low-income urban households or high-income rural

Table 5. Proportions of Chinese children having more than $30 \%$ energy from fat from 1991 to $2009^{\star \star \star} \dagger$

(Mean values with their standard errors)

\begin{tabular}{|c|c|c|c|c|c|c|c|c|c|c|c|c|c|c|}
\hline & \multicolumn{2}{|c|}{1991} & \multicolumn{2}{|c|}{1993} & \multicolumn{2}{|c|}{1997} & \multicolumn{2}{|c|}{2000} & \multicolumn{2}{|c|}{2004} & \multicolumn{2}{|c|}{2006} & \multicolumn{2}{|c|}{2009} \\
\hline & Mean & SE & Mean & $\mathrm{SE}$ & Mean & SE & Mean & SE & Mean & $\mathrm{SE}$ & Mean & SE & Mean & SE \\
\hline All & $20 \cdot 1$ & 3.8 & $25 \cdot 1$ & 3.8 & $34 \cdot 2$ & 3.7 & $46 \cdot 3$ & $3 \cdot 8$ & $46 \cdot 0$ & 3.8 & $44 \cdot 3$ & 3.9 & $49 \cdot 4$ & 3.9 \\
\hline \multicolumn{15}{|c|}{ Age group (years) } \\
\hline $7-10$ & 20.5 & $4 \cdot 3$ & $26 \cdot 1$ & 4.3 & 36.4 & 4.3 & $49 \cdot 2$ & 4.4 & $47 \cdot 3$ & $4 \cdot 6$ & 47.9 & 4.6 & $51 \cdot 8$ & 4.6 \\
\hline $11-13$ & $18 \cdot 2$ & 3.3 & $23 \cdot 3$ & 3.3 & 31.7 & 3.2 & 43.9 & $3 \cdot 2$ & $42 \cdot 2$ & 3.6 & $40 \cdot 9$ & 3.7 & 48.5 & 3.7 \\
\hline $14-17$ & $18 \cdot 2$ & 3.3 & $23 \cdot 2$ & 3.3 & 30.4 & 3.3 & $43 \cdot 1$ & 3.3 & $46 \cdot 6$ & 3.4 & 39.8 & 3.7 & 43.4 & 3.8 \\
\hline \multicolumn{15}{|l|}{ Sex } \\
\hline Male & $18 \cdot 8$ & 3.4 & 24.5 & 3.4 & $32 \cdot 3$ & 3.4 & 43.9 & 3.4 & 47.4 & 3.6 & $42 \cdot 3$ & 3.7 & $48 \cdot 2$ & 3.7 \\
\hline Female & $19 \cdot 1$ & 3.6 & 23.7 & 3.6 & 34.0 & 3.6 & 46.9 & 3.7 & $44 \cdot 0$ & 3.8 & $44 \cdot 2$ & 3.9 & 48.0 & 3.9 \\
\hline \multicolumn{15}{|l|}{ Urban } \\
\hline Low & 22.9 & 5.2 & 29.5 & $5 \cdot 3$ & 40.7 & $5 \cdot 0$ & 57.9 & $5 \cdot 3$ & $46 \cdot 9$ & $5 \cdot 8$ & 54.5 & $6 \cdot 1$ & $66 \cdot 2$ & $6 \cdot 3$ \\
\hline Medium & 35.7 & $5 \cdot 6$ & $47 \cdot 6$ & $5 \cdot 7$ & $59 \cdot 0$ & 5.4 & 68.4 & $5 \cdot 7$ & $67 \cdot 3$ & $6 \cdot 2$ & $60 \cdot 4$ & $6 \cdot 6$ & 62.5 & $6 \cdot 7$ \\
\hline High & 37.7 & $4 \cdot 6$ & $60 \cdot 3$ & 4.7 & $67 \cdot 3$ & 4.5 & $69 \cdot 2$ & 4.7 & $59 \cdot 3$ & 5.5 & 72.9 & 5.7 & $66 \cdot 2$ & $6 \cdot 0$ \\
\hline Total & 32.6 & $4 \cdot 6$ & 46.9 & 4.7 & $56 \cdot 8$ & 4.5 & $67 \cdot 3$ & 4.6 & 58.0 & 4.8 & $64 \cdot 1$ & 4.9 & $66 \cdot 1$ & $5 \cdot 0$ \\
\hline \multicolumn{15}{|l|}{ Rural } \\
\hline Low & 11.6 & 3.2 & $11 \cdot 1$ & 3.2 & $21 \cdot 3$ & 3.2 & $29 \cdot 7$ & $3 \cdot 3$ & $30 \cdot 7$ & 3.5 & 33.3 & 3.7 & 33.3 & 3.7 \\
\hline Medium & 14.4 & 3.8 & $15 \cdot 5$ & 3.9 & 22.4 & 3.8 & 34.5 & 3.9 & $42 \cdot 2$ & $4 \cdot 1$ & $32 \cdot 4$ & 4.3 & $39 \cdot 0$ & 4.3 \\
\hline High & 16.9 & $4 \cdot 3$ & $23 \cdot 2$ & 4.3 & $30 \cdot 1$ & 4.3 & $49 \cdot 8$ & 4.4 & $50 \cdot 0$ & $4 \cdot 6$ & 42.5 & 4.8 & $52 \cdot 6$ & 4.8 \\
\hline Total & $16 \cdot 7$ & 3.9 & $18 \cdot 7$ & 3.9 & $27 \cdot 3$ & 3.9 & 39.9 & 3.9 & 43.0 & 4.0 & 38.3 & 4.0 & $44 \cdot 7$ & 4.0 \\
\hline
\end{tabular}

*** Significant trend in each subgroup across the survey years $(P<0.0001$; test for trend).

$\uparrow$ Values adjusted for age, sex, urban/rural residence and income. 
Table 6. Protein intake (g), by age, sex, residence and income, in Chinese children from 1991 to $2009 \dagger$

(Mean values with their standard errors)

\begin{tabular}{|c|c|c|c|c|c|c|c|c|c|c|c|c|c|c|}
\hline & \multicolumn{2}{|c|}{1991} & \multicolumn{2}{|c|}{1993} & \multicolumn{2}{|c|}{1997} & \multicolumn{2}{|c|}{2000} & \multicolumn{2}{|c|}{2004} & \multicolumn{2}{|c|}{2006} & \multicolumn{2}{|c|}{2009} \\
\hline & Mean & $\overline{\mathrm{SE}}$ & Mean & $\mathrm{SE}$ & Mean & $\mathrm{SE}$ & Mean & $\mathrm{SE}$ & Mean & $\overline{\mathrm{SE}}$ & Mean & $\overline{S E}$ & Mean & $\mathrm{SE}$ \\
\hline All $\|^{\star \star \star}$ & $66 \cdot 2$ & 1.3 & 64.9 & 1.3 & $60 \cdot 1$ & 1.3 & 61.9 & $1 \cdot 3$ & 60.0 & 1.4 & 57.8 & 1.4 & 58.0 & 1.4 \\
\hline \multicolumn{15}{|c|}{ Age group (years) } \\
\hline $7-10^{\star \star \star}$ & $56 \cdot 9$ & $1 \cdot 2$ & 55.9 & $1 \cdot 2$ & 51.5 & $1 \cdot 2$ & 54.4 & 1.3 & $52 \cdot 2$ & 1.4 & 48.4 & 1.4 & 49.9 & 1.4 \\
\hline $11-13^{\star * *}$ & $65 \cdot 8$ & $1 \cdot 6$ & $65 \cdot 0$ & 1.6 & $60 \cdot 8$ & 1.6 & $62 \cdot 2$ & 1.6 & $61 \cdot 0$ & $1 \cdot 8$ & $60 \cdot 4$ & 1.9 & $58 \cdot 0$ & 1.9 \\
\hline $14-17^{\star * \star}$ & $73 \cdot 0$ & 1.3 & 71.5 & 1.4 & 65.4 & 1.4 & $67 \cdot 2$ & 1.4 & 65.9 & 1.5 & $65 \cdot 1$ & 1.7 & 64.5 & 1.8 \\
\hline \multicolumn{15}{|l|}{ Sex } \\
\hline Male $^{\star \star \star}$ & 68.0 & 1.4 & $67 \cdot 6$ & 1.4 & $62 \cdot 1$ & 1.3 & 64.5 & 1.4 & 63.4 & 1.5 & 60.9 & 1.5 & 61.9 & 1.5 \\
\hline Female ${ }^{\star \star \star}$ & $62 \cdot 0$ & 1.2 & $60 \cdot 1$ & $1 \cdot 2$ & 55.9 & $1 \cdot 2$ & 57.4 & $1 \cdot 2$ & $55 \cdot 1$ & 1.3 & 53.3 & 1.4 & 51.8 & 1.4 \\
\hline \multicolumn{15}{|l|}{ Urban } \\
\hline Low $^{\star \star}$ & $62 \cdot 2$ & 2.5 & $62 \cdot 2$ & $2 \cdot 5$ & 61.4 & 2.4 & $62 \cdot 6$ & 2.5 & 60.9 & $2 \cdot 7$ & 55.4 & 2.9 & 58.4 & 2.9 \\
\hline Medium & $66 \cdot 1$ & $2 \cdot 8$ & 67.5 & $2 \cdot 8$ & $65 \cdot 8$ & $2 \cdot 7$ & 64.5 & $2 \cdot 8$ & $66 \cdot 5$ & 3.0 & 59.8 & 3.1 & $66 \cdot 2$ & $3 \cdot 2$ \\
\hline High & $67 \cdot 1$ & $2 \cdot 8$ & 68.4 & $2 \cdot 8$ & $69 \cdot 1$ & $2 \cdot 7$ & $71 \cdot 1$ & $2 \cdot 8$ & 70.9 & $3 \cdot 1$ & 68.4 & 3.2 & 67.2 & $3 \cdot 3$ \\
\hline All ${ }^{\star \star \star \star}$ & 65.6 & 2.5 & 66.6 & 2.5 & $66 \cdot 1$ & 2.5 & 66.9 & 2.5 & 65.4 & $2 \cdot 6$ & $61 \cdot 2$ & $2 \cdot 6$ & $64 \cdot 3$ & $2 \cdot 7$ \\
\hline \multicolumn{15}{|l|}{ Rural } \\
\hline Low $^{\star \star \star}$ & $63 \cdot 1$ & 1.4 & $60 \cdot 2$ & 1.4 & 53.7 & 1.5 & 52.7 & 1.5 & $50 \cdot 1$ & 1.7 & 53.3 & 1.8 & 48.7 & 1.8 \\
\hline Medium $^{\star \star *}$ & $63 \cdot 9$ & 1.5 & $63 \cdot 3$ & 1.5 & $55 \cdot 6$ & 1.5 & $58 \cdot 3$ & 1.5 & 58.0 & 1.7 & 52.9 & $1 \cdot 8$ & 54.4 & 1.8 \\
\hline $\mathrm{High}^{\star \star \star}$ & $67 \cdot 0$ & 1.5 & $65 \cdot 1$ & 1.5 & 59.6 & 1.5 & $65 \cdot 1$ & 1.6 & $62 \cdot 3$ & 1.7 & $61 \cdot 1$ & 1.8 & 59.4 & 1.8 \\
\hline All ${ }^{\star \star \star \star}$ & $66 \cdot 3$ & 1.5 & $64 \cdot 2$ & 1.5 & $57 \cdot 8$ & 1.5 & $60 \cdot 1$ & 1.5 & 58.2 & 1.6 & 56.9 & 1.6 & 55.9 & 1.6 \\
\hline
\end{tabular}

Significant trend across the survey years: ${ }^{* *} P<0.05,{ }^{* * *} P<0.0001$; test for trend

† Values adjusted for age, sex, urban/rural residence and income.

households. These findings suggest that future disease burdens related to this nutritional transition may shift from high-income urban populations to medium-to-low-income urban and high-income rural populations.

\section{Energy intake}

Our analysis found that energy intake in Chinese children dropped in the last two decades. This trend is consistent with that observed in US children from $9920 \mathrm{~kJ}$ in 1965 to $8770 \mathrm{~kJ}$ in $1990^{(6-9,11)}$, although this decline was subsequently followed by an increase to reach $9580 \mathrm{~kJ}$ in $1995^{(6,8-11)}$. As a result, the energy intake in Chinese children was higher than that in US children in 1990, but it was reversed by 1995 . Further, the decline in Chinese children during this period was much faster than that in US children between 1965 and $1990^{(6,8,9)}$. The more rapid transition in Chinese children might be attributed to the accelerated introduction of technology into their daily life activities ${ }^{(2)}$. Considering the increasing prevalence of child obesity in China ${ }^{(3)}$, the decline in energy intake may be a response to the decline in physical activity ${ }^{(6)}$ as reported in our previous analysis that both passive commuting to school $^{(22)}$ and sedentary behaviours ${ }^{(23)}$ increased in Chinese children during the last decade. Thus, the larger decline in energy intake for children in rural areas or from lower-income households might be attributed to a larger decline in their level of physical activity ${ }^{(22)}$.

There is limited information about the nutrition transition in children in developing countries. In contrast to our findings, children in the metropolitan Cebu region in the Philippines experienced an increase in daily energy intake from $5018 \mathrm{~kJ}$ in 1994 to $7990 \mathrm{~kJ}$ in $2002^{(10)}$. This inconsistency might derive from the different stages of economic development in the two countries. In 1994, the Philippines was still recovering from the 1991 economic recession, which was followed by a period of economic development. In contrast, China's gross domestic product (GDP) has been steadily increasing at an average of $9.4 \%$ per year from 1980 to $2002^{(24)}$. Consistent with data from adults in China ${ }^{(5,25)}$ and most other developing countries ${ }^{(13,16,26-31)}$, there has been a dramatic decline in energy intake in parallel with economic development, except in Malaysia(17), Morocco $^{(14)}$ and Chile ${ }^{(15)}$, where energy intake increased during the last four decades in the last century. These inconsistencies might be attributed to different methods for the estimation of dietary intake and the different stages of economic development in the time periods examined between the data from these three countries and CHNS.

\section{Carbohydrate intake}

Our analysis found a continuous decline in the intake of carbohydrate and the proportion of energy from carbohydrate during the last two decades in Chinese youth. Carbohydrate is the main source of dietary energy in the traditional diet of Chinese children. The decline in carbohydrate intake, and thus dietary energy intake, is a reflection of the drop in energy expenditure as lifestyles have become more sedentary ${ }^{(32)}$. In contrast to our findings, carbohydrate intake of US adolescents decreased from $274 \mathrm{~g}$ in 1965 to $247 \mathrm{~g}$ in 1977 followed by an increase to $269 \mathrm{~g}$ in 1990 and to $310 \mathrm{~g}$ in 1995, while the proportion of energy from carbohydrate steadily increased between 1965 and 1996(6). The decline in both carbohydrate intake and percentage of dietary energy from carbohydrate in Chinese youth was similar to that observed in adults in China ${ }^{(5)}$, in other Asian countries ${ }^{(17,28-31)}$ and in Mexico ${ }^{(16)}$, while inconsistent with adults in Chile ${ }^{(15)}$ and Morocco ${ }^{(14)}$, where carbohydrate intake increased, resulting in an increase in energy intake from 1960 to 2000. 


\section{Fat intake}

Fat intake and the proportion of energy from fat increased in Chinese youth during the last two decades. Contrary to our findings, the consumption of fat in US youth decreased from a very high fat intake of $102 \mathrm{~g}$ in 1965 to $80 \mathrm{~g}$ in 1990 and then increased to $83 \mathrm{~g}$ in 1995, while there was a steady decline in the proportion of energy from fat from 38.7 to $32 \cdot 7 \%$ in US youth between 1965 and $1996^{(6,7)}$. The increase in the consumption of fat and proportion of energy from fat in Chinese youth is consistent with that in adults worldwide experiencing economic development and nutrition transition in the last four decades of the last century $^{(5,14-17,28,30,31)}$. Surprisingly, although experiencing an increase in both fat intake and proportion of energy from fat, South Koreans still adhere to traditional dietary patterns with a low fat intake and low proportion of energy from fat, which might partly be attributed to movements to retain the traditional diet in South Korea ${ }^{(30)}$. The proportion of energy from fat in South Koreans was even lower than that concurrently in the Chinese although China was less developed than South Korea ${ }^{(30)}$. This suggests that a traditional diet can be maintained during economic development if proper measures are taken.

\section{Protein}

Animal food is an important source of dietary fat. The increase in fat intake may partly be driven by increases in animal food in our present study. Surprisingly, protein in the children's diets decreased in the last two decades. A possible explanation is that the decline in protein intake was due to dramatically reduced plant food intake that was greater than the increased intake of protein from animal foods.

\section{Dietary pattern transition}

The traditional dietary pattern in China conforms to the lowfat and high-carbohydrate diet type. Our study found that the traditional Chinese diet has been shifting to a dietary pattern linked with non-communicable diseases ${ }^{(1)}$. The proportions of energy from carbohydrate, fat and protein in Chinese children $(56 \cdot 8,30$ and $13 \cdot 1 \%$, respectively) in 2009 were very close to those $(54 \cdot 2,32 \cdot 7$ and $14 \cdot 2 \%$, respectively) in US adolescents in $1995^{(6)}$. Further, the proportion of children who consumed a diet with more than $30 \%$ of energy from fat more than doubled since 1991 to approximately $50 \%$ in 2009. These findings suggest that the burden of future nutrition-related chronic diseases will increase in this population, considering that energy expenditure is decreasing at the same time, if no effective interventions to prompt healthy diets are taken immediately. These measures are particularly needed in urban areas where, on average, more than one-third of energy was from fat and approximately two-thirds of children consumed a diet with more than $30 \%$ of energy from fat.

Our analysis found that the most dramatic transition in diet patterns took place among children who come from low-income urban households, or high-income rural households. The daily intake of fat increased by $10.8 \mathrm{~g}$ in children from low-income urban households and $24 \mathrm{~g}$ in those from high-income rural households during the last two decades. As a result, the proportion of children who consumed a high-fat diet increased by $43.3 \%$ (from 22.9 to $66.2 \%$ ) in children from low-income urban households, and 35.7\% (from 16.9 to $52.6 \%$ ) in those from high-income rural households during the same period, respectively. This dramatic nutrition transition may lead to a future dramatic increase in non-communicable diseases in these populations ${ }^{(1,4)}$.

\section{Strengths and limitations}

One of the strengths of our study was the individual consecutive $3 \mathrm{~d}$ dietary recall methods that were identical across surveys. The identical dietary measurements across surveys and the short interval between surveys allowed us to carefully examine the temporal trends in dietary intake. Further, the careful data quality control methods may have reduced the misreports of dietary intake, by comparing the individual's average daily dietary intake from the $3 \mathrm{~d}$ diet recall data with that calculated from the household food weighing surveys. One of the limitations of this study was that the effect of using parents as a proxy to report the dietary consumption of young children is unknown. In addition, improvements in food composition databases and actual changes in food composition must be considered in the interpretation of trends in the intake of energy and macronutrients. Also, as the obese are more likely to under-report their dietary intake than normal-weight individuals ${ }^{(33)}$, the average level of underreporting may have increased with the increase in the prevalence of child obesity in China during the study period ${ }^{(3)}$.

\section{Conclusion}

Chinese children and adolescents have been undergoing a rapid nutrition transition to a high-fat diet, which was faster than that observed in Western children in the last century ${ }^{(2)}$. This nutrition transition may be linked with a future increase in chronic non-communicable diseases in this population which has limited access to medical resources. In future studies, special attention should be paid to children who come from low-income urban or high-income rural households who may have been undergoing faster nutrition transition than their peers from high-income urban or low-income rural households.

\section{Acknowledgements}

The authors thank the National Institute of Nutrition and Food Safety, China Center for Disease Control and Prevention, Carolina Population Center, the University of North Carolina at Chapel Hill, the National Institutes of Health (NIH) (R01HD30880, DK056350 and R01-HD38700) and the Fogarty International Center, NIH for financial support for the CHNS data collection and analysis files. The authors also thank the Australian Agency for International Development (AusAID) 
for funding Zhaohui Cui's doctoral scholarship in International Public Health at the University of Sydney, Australia, and this analysis, which is part of the requirements for his PhD studies. Z. C. analysed and interpreted the data, and wrote the manuscript. M. J. D. contributed to writing of the manuscript and data interpretation, and had primary responsibility for the final content. All authors approved the final version of the manuscript. The authors declare that they have no conflicts of interest.

\section{References}

1. Popkin BM (2006) Global nutrition dynamics: the world is shifting rapidly toward a diet linked with noncommunicable diseases. Am J Clin Nutr 84, 289-298.

2. Popkin BM (2002) The shift in stages of the nutrition transition in the developing world differs from past experiences! Public Health Nutr 5, 205-214.

3. Cui Z, Huxley R, Wu Y, et al. (2010) Temporal trends in overweight and obesity of children and adolescents from nine provinces in China from 1991-2006. Int I Pediatr Obes 5, 365-374.

4. Popkin BM \& Du S (2003) Dynamics of the nutrition transition toward the animal foods sector in China and its implications: a worried perspective. J Nutr 133, 3898S-3906S.

5. Zhai F, Wang H, Du S, et al. (2007) Lifespan nutrition and changing socio-economic conditions in China. Asia Pac J Clin Nutr 16, Suppl. 1, 374-382.

6. Cavadini C, Siega-Riz AM \& Popkin BM (2000) US adolescent food intake trends from 1965 to 1996. Arch Dis Child 83, $18-24$.

7. Troiano RP, Briefel RR, Carroll MD, et al. (2000) Energy and fat intakes of children and adolescents in the United States: data from the National Health and Nutrition Examination Surveys. Am J Clin Nutr 72, 1343S-1353S.

8. Nielsen SJ, Siega-Riz AM \& Popkin BM (2002) Trends in energy intake in U.S. between 1977 and 1996: similar shifts seen across age groups. Obes Res 10, 370-378.

9. Briefel RR \& Johnson CL (2004) Secular trends in dietary intake in the United States. Annu Rev Nutr 24, 401-431.

10. Adair LS \& Popkin BM (2005) Are child eating patterns being transformed globally? Obes Res 13, 1281-1299.

11. Kennedy ET, Bowman SA \& Powell R (1999) Dietary-fat intake in the US population. $J$ Am Coll Nutr 18, 207-212.

12. Popkin BM (1994) The nutrition transition in low-income countries: an emerging crisis. Nutr Rev 52, 285-298.

13. Madanat HN, Troutman KP \& Al-Madi B (2008) The nutrition transition in Jordan: the political, economic and food consumption contexts. Promot Educ 15, 6-10.

14. Benjelloun S (2002) Nutrition transition in Morocco. Public Health Nutr 5, 135-140.
15. Albala C, Vio F, Kain J, et al. (2002) Nutrition transition in Chile: determinants and consequences. Public Health Nutr 5, 123-128.

16. Rivera JA, Barquera S, Campirano F, et al. (2002) Epidemiological and nutritional transition in Mexico: rapid increase of non-communicable chronic diseases and obesity. Public Health Nutr 5, 113-122.

17. Noor MI (2002) The nutrition and health transition in Malaysia. Public Health Nutr 5, 191-195.

18. Chanmugam P, Guthrie JF, Cecilio S, et al. (2003) Did fat intake in the United States really decline between 1989-1991 and 1994-1996? J Am Diet Assoc 103, 867-872.

19. Harnack LJ, Jeffery RW \& Boutelle KN (2000) Temporal trends in energy intake in the United States: an ecologic perspective. Am J Clin Nutr 71, 1478-1484.

20. Popkin BM, Keyou G, Zhai F, et al. (1993) The nutrition transition in China: a cross-sectional analysis. Eur I Clin Nutr 47, 333-346.

21. Wang Y, Popkin B \& Zhai F (1998) The nutritional status and dietary pattern of Chinese adolescents, 1991 and 1993. Eur J Clin Nutr 52, 908-916.

22. Cui Z, Bauman A \& Dibley MJ (2011) Temporal trends and correlates of passive commuting to and from school in children from 9 provinces in China. Prev Med 52, 423-427.

23. Cui Z, Hardy LL, Dibley MJ, et al. (2011) Temporal trends and recent correlates in sedentary behaviours in Chinese children. Int J Behav Nutr Phys Act 8, 93

24. International Monetary Fund (2011) World Economic Outlook Database. http://www.imf.org/external/pubs/ft/weo/ 2011/01/weodata/index.aspx (accessed May 2011).

25. Du S, Lu B, Zhai F, et al. (2002) A new stage of the nutrition transition in China. Public Health Nutr 5, 169-174

26. Galal OM (2002) The nutrition transition in Egypt: obesity, undernutrition and the food consumption context. Public Health Nutr 5, 141-148

27. Lee SK \& Sobal J (2003) Socio-economic, dietary, activity, nutrition and body weight transitions in South Korea Public Health Nutr 6, 665-674

28. Kosulwat V (2002) The nutrition and health transition in Thailand. Public Health Nutr 5, 183-189.

29. Shetty PS (2002) Nutrition transition in India. Public Health Nutr 5, 175-182.

30. Kim S, Moon S \& Popkin BM (2000) The nutrition transition in South Korea. Am J Clin Nutr 71, 44-53.

31. Lipoeto NI, Wattanapenpaiboon N, Malik A, et al. (2004) Nutrition transition in west Sumatra, Indonesia. Asia Pac J Clin Nutr 13, 312-316.

32. Popkin BM (2001) Nutrition in transition: the changing global nutrition challenge. Asia Pac J Clin Nutr 10, S13-S18.

33. Livingstone MB \& Black AE (2003) Markers of the validity of reported energy intake. J Nutr 3, Suppl. 3, 895S-920S. 